HIP-2008-07/TH

NSF-KITP-08-10

\title{
The diagonal and off-diagonal quark number susceptibility of high temperature and finite density QCD
}

\author{
A. Hietanen ${ }^{\mathrm{a}}$, K. Rummukainen ${ }^{\mathrm{b}}$, \\ a Theoretical Physics Division, Department of Physics \\ and Helsinki Institute of Physics, \\ P.O.Box 64, FI-00014 University of Helsinki, Finland \\ ${ }^{\mathrm{b}}$ Department of Physics, \\ University of Oulu P.O.Box 3000, FI-90014 Oulu, Finland
}

\begin{abstract}
We study the quark number susceptibility of the hot quark-gluon plasma at zero and non-zero quark number density, using lattice Monte Carlo simulations of an effective theory of QCD, electrostatic QCD (EQCD). Analytic continuation is used to obtain results at non-zero quark chemical potential $\mu$. We measure both flavor singlet (diagonal) and non-singlet (off-diagonal) quark number susceptibilities. The diagonal susceptibility approaches the perturbative result above $\sim 20 T_{\mathrm{c}}$, but below that temperature we observe significant deviations. The results agree well with $4 \mathrm{~d}$ lattice data down to temperatures $\sim 2 T_{\mathrm{c}}$. The off-diagonal susceptibility is more prone to statistical and systematic errors, but the results are consistent with perturbation theory already at $10 T_{\mathrm{c}}$.
\end{abstract}




\section{Introduction}

The quark (baryon) number susceptibility of hot QCD matter characterizes the "softness" of the equation of state. It is directly related to the event-by-event fluctuations observed in heavy ion collision experiments [1, probing the phase diagram and the properties of the hot QCD plasma. Thus, it is of significant interest to calculate it theoretically as accurately as possible. Hence, several calculations of susceptibility have been published using lattice simulations [2, 3, 4, 5, 6, 7, 8, or perturbation theory [9, 10, 11].

In this work we use lattice Monte Carlo simulations in order to measure the diagonal (flavor singlet) and off-diagonal (non-singlet) quark number susceptibilities at high temperatures and at non-zero densities. Instead of full 4-dimensional QCD, the theory we study on the lattice is a dimensionally reduced effective theory of the hot quark-gluon plasma phase of QCD, electrostatic QCD (EQCD) [12, 13, 14, 15, 16]. It is by now well established that EQCD can accurately describe many properties of the hot QCD plasma, and it provides a very convenient starting point for studying high-temperature QCD using perturbative analysis [17, 18, 19] or non-perturbative lattice simulations.

The validity of the effective theory approach is based on the fact that at high enough temperatures the gauge coupling constant $g$ becomes small, giving rise to three relevant momentum scales (neglecting quark masses): hard scale $p \sim \pi T$, corresponding to non-zero Matsubara frequencies, soft electric scale $\sim g T$ and supersoft magnetic scale $\sim g^{2} T$. EQCD is obtained by (formally) integrating over the hard scales perturbatively, leaving an effective theory for soft and supersoft scales. All infrared divergences inherent in finite temperature field theories are correctly contained in the effective theory. A crucial feature of EQCD is that all of the fermionic modes are integrated over, leaving a purely bosonic theory.

EQCD offers an interesting alternative to standard high-temperature lattice simulations. Above all, the theory is three-dimensional and purely bosonic, making it much cheaper to simulate. The standard QCD lattice simulations work well at temperatures up to 5-10 $T_{\mathrm{c}}$, but due to the sheer cost of the simulations with light quarks it can be very difficult to obtain accurate results. In contrast the perturbative analysis works at temperatures $T \gtrsim 10 T_{\mathrm{c}}$ (albeit with slow convergence), but since the infrared singularities in the magnetic sector cannot be treated perturbatively the accuracy is limited to some order (depending on the observable) in the coupling constant expansion. The lattice simulations of EQCD fully include the effects of the infrared singularities, thus offering a clear way to improve on the perturbative results. While EQCD cannot describe the QCD phase transition, it has been observed to be quantitatively accurate down to temperatures of order 2-4 $T_{c}$, depending on the quantity of interest. On the other hand, it is relatively easy to do EQCD simulations at arbitrarily high temperatures, enabling one to quantify the convergence to the perturbation theory and the role of the infrared singularities. Lattice simulations of EQCD have been used to calculate QCD pressure at high temperature [20, 21], spatial string tension [22], and spatial screening lengths [23, 24, 25]. 
In this paper we present the lattice calculations using EQCD to measure the diagonal and off-diagonal quark number (baryon number) susceptibilities at zero and non-zero baryon chemical potential. At non-zero chemical potential EQCD suffers from a sign problem, albeit this is milder than in full QCD. The finite chemical potential results are obtained by performing simulations with imaginary values of the chemical potential and then analytically continuing to real chemical potential. We observe that the deviations from the perturbation theory are significant up to temperatures of order $20 T_{\mathrm{c}}$. On the other hand, EQCD is observed to work at surprisingly low temperatures: our results agree well with existing $4 \mathrm{~d}$ lattice simulations even slightly below $2 T_{\mathrm{c}}$. The method also is well suited for simulations at non-zero chemical potential, because our observations agree those of [5] and extend to even higher values of chemical potential. The results have been partly published in [26, 27].

The paper is organised as follows. In Sec. 2 we give the theoretical background and specify the considered observables. In Sec. 3 we present the numerical results of lattice Monte Carlo Simulations. Conclusions are given in Sec. 4 .

\section{Effective theory}

\subsection{Action}

The electrostatic QCD with finite chemical potential $\mu$ is defined by the action

$$
\begin{aligned}
S_{\mathrm{E}} & =\int \mathrm{d}^{3} x \mathcal{L}_{\mathrm{E}} \\
\mathcal{L}_{\mathrm{E}} & =\frac{1}{2} \operatorname{Tr}\left[F_{i j}^{2}\right]+\operatorname{Tr}\left[D_{i}, A_{0}\right]^{2}+m_{3}^{2} \operatorname{Tr}\left[A_{0}^{2}\right]+i \gamma_{3} \operatorname{Tr}\left[A_{0}^{3}\right]+\lambda_{3}\left(\operatorname{Tr}\left[A_{0}^{2}\right]\right)^{2},
\end{aligned}
$$

where $F_{i j}=\partial_{i} A_{j}-\partial_{j} A_{i}+i g_{3}\left[A_{i}, A_{j}\right]$ and $D_{i}=\partial_{i}+i g_{3} A_{i} . \quad F_{i j}, A_{i}$ and $A_{0}$ are traceless $3 \times 3$ Hermitean matrices $\left(A_{0}=A_{0}^{a} T_{a}\right.$, etc). The theory has 4 parameters: $g_{3}^{2}$ (3-dimensional gauge coupling), $m_{3}^{2}, \lambda_{3}$ and $\gamma_{3}$, with dimensions $\left[g_{3}^{2}\right]=\left[\lambda_{3}\right]=\mathrm{GeV},\left[\gamma_{3}\right]=\mathrm{GeV}^{3 / 2}$ and $\left[m_{3}^{2}\right]=\mathrm{GeV}^{2}$. Non-zero value of the parameter $\gamma_{3}$, caused by non-zero quark chemical potential, renders the action complex. Thus, this theory is not free from the sign problem of finite density QCD.

It is convenient to define three dimensionless ratios

$$
y=\frac{m_{3}^{2}}{g_{3}^{4}}, \quad x=\frac{\lambda_{3}}{g_{3}^{2}}, \quad z=\frac{\gamma_{3}}{g_{3}^{3}},
$$

leaving only $g_{3}^{2}$ dimensionful. Through the dimensional reduction process (perturbative matching of suitable observables in EQCD and real QCD), the parameters of EQCD become functions of physical 4d parameters: the temperature $T$ and the chemical potential $\mu$ (the quark masses are set to zero). The parameters are also functions of the renormalization scale $\Lambda_{\overline{\mathrm{MS}}}$ used in the derivation of the effective theory. If we denote the number of quark 
flavors by $N_{\mathrm{f}}$, for $N_{\mathrm{c}}=3$ the relations are [16, 24]:

$$
\begin{aligned}
g_{3}^{2}= & \frac{24 \pi^{2}}{33-2 N_{\mathrm{f}}} \frac{T}{\bar{\Lambda}_{g} / \Lambda_{\overline{\mathrm{MS}}}}\left(1-\sum_{i=1}^{N_{\mathrm{f}}} \frac{1}{9-N_{\mathrm{f}}} \mathcal{D}\left(\bar{\mu}_{i}\right) x+\mathcal{O}\left(x^{2}\right)\right) \\
x= & \frac{9-N_{\mathrm{f}}}{33-2 N_{\mathrm{f}}} \frac{1}{\bar{\Lambda}_{x} / \Lambda_{\overline{\mathrm{MS}}}}\left(1-\sum_{i=1}^{N_{\mathrm{f}}} \frac{1}{9-N_{\mathrm{f}}} \mathcal{D}\left(\bar{\mu}_{i}\right) x+\mathcal{O}\left(x^{2}\right)\right) \\
y= & \frac{\left(9-N_{\mathrm{f}}\right)\left(6+N_{\mathrm{f}}\right)}{144 \pi^{2} x}\left(1+\sum_{i=1}^{N_{\mathrm{f}}} \frac{3}{6+N_{\mathrm{f}}} \bar{\mu}_{i}^{2}\right)+ \\
& \frac{486-33 N_{\mathrm{f}}-11 N_{\mathrm{f}}^{2}-2 N_{\mathrm{f}}^{3}}{96 \pi^{2}\left(9-N_{\mathrm{f}}\right)}\left(1+\sum_{i=1}^{N_{\mathrm{f}}} \frac{3\left(7+N_{\mathrm{f}}\right)\left(9-2 N_{\mathrm{f}}\right)}{486-33 N_{\mathrm{f}}-11 N_{\mathrm{f}}^{2}-2 N_{\mathrm{f}}^{3}} \bar{\mu}_{i}^{2}\right)+\mathcal{O}(x) \\
z= & \sum_{i=1}^{N_{\mathrm{f}}} \frac{\bar{\mu}_{i}}{3 \pi}\left(1+\frac{21+3 N_{\mathrm{f}}}{18-2 N_{\mathrm{f}}} x\right)+\mathcal{O}\left(x^{2}\right),
\end{aligned}
$$

where $\bar{\mu}=\mu /(\pi T)$, and, for small $\bar{\mu}, \mathcal{D}(\bar{\mu}) \approx-7 \zeta(3) \bar{\mu}^{2} / 2$, and

$$
\begin{aligned}
& \bar{\Lambda}_{g}=4 \pi T \exp \left(\frac{-3+4 N_{\mathrm{f}} \log 4}{66-4 N_{\mathrm{f}}}-\gamma_{\mathrm{E}}\right), \\
& \bar{\Lambda}_{x}=4 \pi T \exp \left(\frac{-162+102 N_{\mathrm{f}}-4 N_{\mathrm{f}}^{2}+\left(36 N_{\mathrm{f}}-4 N_{\mathrm{f}}^{2}\right) \log (4)}{594-75 N_{\mathrm{f}}+N_{\mathrm{f}}^{2}}-\gamma_{\mathrm{E}}\right) .
\end{aligned}
$$

The dimensional reduction scheme is expected to be valid temperatures down to $\sim 2-4 T_{c}$ and chemical potential up to $\mu \sim \pi T$ or $\bar{\mu} \sim 1$. For $N_{\mathrm{f}}=2$ these values correspond to $x \sim 0.1$ and $z \sim 0.1$. At higher temperatures $x$ becomes rapidly smaller. Hence, the higher order corrections in $x$ in above formulas become in practice very small, and we ignore corrections $\mathcal{O}(x)$ in above expressions. We shall further restrict ourselves to 2 massless quarks, $N_{\mathrm{f}}=2$ :

$$
\begin{aligned}
g_{3}^{2} & =\left.g_{3}^{2}\right|_{\mu=0} \\
x & =\left.x\right|_{\mu=0} \\
y & =\left.y\right|_{\mu=0}\left(1+\frac{3}{8} \sum_{i=1}^{N_{\mathrm{f}}} \bar{\mu}_{i}^{2}\right) \equiv y_{0}\left(1+\frac{3}{8} \sum_{i=1}^{N_{\mathrm{f}}} \bar{\mu}_{i}^{2}\right) \\
z & =\sum_{i=1}^{N_{\mathrm{f}}} \frac{\bar{\mu}_{i}}{3 \pi} .
\end{aligned}
$$

See [24] for more discussion about the effect of this approximation.

\subsection{Susceptibility}

We define the quark number susceptibility in EQCD as:

$$
\chi_{3, i j}=\frac{1}{V} \frac{\partial^{2}}{\partial \bar{\mu}_{i} \partial \bar{\mu}_{j}} \ln \mathcal{Z}=\frac{1}{V} \frac{\partial^{2}}{\partial \bar{\mu}_{i} \partial \bar{\mu}_{j}} \ln \int \mathcal{D} A_{k} \mathcal{D} A_{0} \exp \left(-S_{\mathrm{E}}\right),
$$


where $i, j$ stands for quark flavors $\mathrm{u}$ and $\mathrm{d}$, and label 3 indicates that this is a result from 3-dimensional effective theory. Thus, there are two independent components of the susceptibility: diagonal $(i=j)$ and off-diagonal $(i \neq j)$. Using the shorthand notation for the dimensionless volume averages

$$
\hat{A}_{0}^{n} \equiv \frac{1}{g_{3}^{n} V} \int d^{3} x \operatorname{Tr} A_{0}^{n}(x)
$$

and defining the condensates

$$
\begin{aligned}
& C_{1}=\left\langle\hat{A}_{0}^{2}\right\rangle \\
& C_{2}=V g_{3}^{6}\left(\left\langle\left(\hat{A}_{0}^{3}\right)^{2}\right\rangle-\left\langle\hat{A}_{0}^{3}\right\rangle^{2}\right) \\
& C_{3}=V g_{3}^{6}\left(\left\langle\left(\hat{A}_{0}^{2}\right)^{2}\right\rangle-\left\langle\hat{A}_{0}^{2}\right\rangle^{2}\right) \\
& C_{4}=V g_{3}^{6}\left(\left\langle\hat{A}_{0}^{3} \hat{A}_{0}^{2}\right\rangle-\left\langle\hat{A}_{0}^{3}\right\rangle\left\langle\hat{A}_{0}^{2}\right\rangle\right),
\end{aligned}
$$

we can write the susceptibility as

$$
\frac{\chi_{3, i j}}{g_{3}^{6}}=-\frac{3}{4} \delta_{i j} y_{0} C_{1}-\frac{1}{9 \pi^{2}} C_{2}+\frac{9}{16} \bar{\mu}_{i} \bar{\mu}_{j} y_{0}^{2} C_{3}+i \frac{1}{4 \pi}\left(\bar{\mu}_{i}+\bar{\mu}_{j}\right) y_{0} C_{4}
$$

We note here the rather striking fact that the expectation value in $C_{4}$ is purely imaginary for real $\bar{\mu}$, rendering the full expression real. The imaginary expectation value comes from the complex measure; $\hat{A}_{0}^{3}$ and $\hat{A}_{0}^{2}$ itself are always real-valued.

\subsection{Analytic continuation}

The sign problem of finite density QCD is manifested here as an imaginary term in the EQCD action, Eq. (2.1). This makes the standard Monte Carlo importance sampling impractical, except for very small chemical potentials and/or small volumes. One option to circumvent this problem is to use analytic continuation to complex values of $\bar{\mu}$ : the sign problem vanishes for purely imaginary $\bar{\mu}$.

However, we emphasize that the direct analytic continuation in $\bar{\mu}$ is clearly suboptimal and unnecessary in this case: of the terms appearing in EQCD action Eq. (2.1), only $i \gamma_{3} \operatorname{Tr}\left[A_{0}^{3}\right]$ is responsible for the sign problem. Thus, it is sufficient to analytically continue $\gamma_{3}$ (or $z$ ) to imaginary values and leave the other parameters to the values determined by the desired value of $\bar{\mu}$. By far the dominant effect of non-zero $\bar{\mu}$ is due to the $\bar{\mu}$-dependence of the parameter $y$ in Eq. (2.9), we can take into account almost all of the effects of the chemical potential by just using the correct $y(\bar{\mu})$. The remaining small corrections are then taken into account by analytic continuation $z \rightarrow i z, 1$

\footnotetext{
${ }^{1}$ In [26] the susceptibility was evaluated by ignoring this correction; the improved statistics here make the small correction non-negligible.
} 
Because the action (2.1) is invariant under the simultaneous change $z \rightarrow-z$ and $A_{0} \rightarrow-A_{0}$, the partition function must be an even function of $z$ (and $\mu$ ). From this follows that the expectation values $\left\langle\hat{A}_{0}^{n}\right\rangle$ are even (odd) functions of $z$ for even (odd) $n$. Therefore, we can Taylor expand the condensates $C_{i}$ appearing in the expression for the susceptibility (2.13) in powers of $z$ as appropriate:

$$
C_{i}(z)=\sum_{n} c_{i, n} z^{n}=\sum_{n} i^{n} c_{i, n}(-i z)^{n}
$$

The analytic continuation now proceeds as follows: we perform simulations with imaginary value of $z$ and determine the Taylor series coefficients $c_{i, n}$ for each of the condensates up to the desired order. Using Eq. (2.14) we obtain the the condensates $C_{i}$ at real values of $z$, which can be inserted in Eq. (2.13) in order to obtain the susceptibility.

The dependence of the condensates on $z$ is very mild, as expected, and it turns out to be sufficient to expand the condensates to very low order:

$$
\begin{array}{ll}
C_{1}=a_{1}+a_{2} z^{2} & C_{3}=a_{4} \\
C_{2}=a_{3} & C_{4}=\frac{\partial C_{1}}{\partial(i z)}=-2 i a_{2} z .
\end{array}
$$

Note that we assume that $C_{2}$ and $C_{3}$ are independent of $z$. This is indeed the case to the statistical accuracy we can reach.

If we now denote with $C_{i}\left(z_{\mathrm{I}}\right)$ the condensates measured from simulations with imaginary $z=\left(0, z_{\mathrm{I}}\right)$, the susceptibility at real $z=\left(z_{\mathrm{R}}, 0\right)$ becomes

$$
\begin{aligned}
\frac{\chi_{3, i j}\left(z_{\mathrm{R}}\right)}{g_{3}^{6}} & =-\frac{3}{4} \delta_{i j} y_{0}\left(C_{1}\left(z_{\mathrm{I}}\right)+\frac{z_{\mathrm{R}}^{2}}{z_{\mathrm{I}}} C_{4}\left(z_{\mathrm{I}}\right)\right)-\frac{1}{9 \pi^{2}} C_{2}\left(z_{\mathrm{I}}\right) \\
& +\frac{9}{16} \bar{\mu}_{i} \bar{\mu}_{j} y_{0}^{2} C_{3}\left(z_{\mathrm{I}}\right)+\frac{1}{4 \pi}\left(\bar{\mu}_{i}+\bar{\mu}_{j}\right) y_{0} \frac{z_{\mathrm{R}}}{z_{\mathrm{I}}} C_{4}\left(z_{\mathrm{I}}\right) .
\end{aligned}
$$

We note here that one simulation at some $z_{\mathrm{I}}$ is sufficient to obtain the condensates and the susceptibility at all (small enough) $z_{\mathrm{R}}$. However, because both $y$ and $z$ depend on $\bar{\mu}$, only the value of $z_{\mathrm{R}}$ which corresponds to $\bar{\mu}$ used in evaluating $y$ is physical. Thus, for each value of the chemical potential we need to do a new simulation. We also choose to use $z_{\mathrm{I}}=z_{\mathrm{R}}$ in our simulations, eliminating the ratios $z_{\mathrm{I}} / z_{\mathrm{R}}$ in Eq. (2.16). In what follows we shall use the notation $z=z_{\mathrm{R}}=z_{\mathrm{I}}$ to refer to both quantities.

The phase diagram of EQCD has 3 distinct phases: a symmetric phase with $\left\langle\hat{A}_{0}^{3}\right\rangle=0$ and 2 broken phases with non-zero $\left\langle\hat{A}_{0}^{3}\right\rangle$, related by reflection $\left\langle\hat{A}_{0}^{3}\right\rangle \leftrightarrow-\left\langle\hat{A}_{0}^{3}\right\rangle$ [28]. In order to properly represent $4 \mathrm{~d} \mathrm{QCD}$, the effective theory must remain in the symmetric phase. In the absence of the chemical potential the symmetric phase is at most metastable, when the parameters $x$ and $y$ are fixed to values which correspond to $4 \mathrm{~d}$ QCD. This is normally not a problem, because the metastability is very strong and for all practical purposes the symmetric phase remains stable. 
Applying imaginary chemical potential to the full action would decrease the value of the parameter $y(\mu)$, Eq. (2.9) . Hence, the metastability would be reduced and finally completely lost at some value of imaginary $\mu$. However, for our method of analytic continuation this problem is completely avoided: because we calculate $y(\mu)$ with real $\mu$, the value of $y$ increases as $\mu$ increases. Thus, the physical symmetric phase remains stable at all values of $\mu$.

\subsection{Relation to $4 \mathrm{~d}$ physics}

The relation between $\chi_{3, i j}$ and the physical $4 \mathrm{~d}$ susceptibility is given by

$$
\frac{\chi_{i j}}{T^{2}}=\frac{g_{3}^{6}}{\pi^{2} T^{3}} \chi_{3, i j}+\frac{\partial^{2}}{\partial \mu_{i} \partial \mu_{j}} \Delta p,
$$

where $\Delta p=p_{\mathrm{QCD}}-p_{\mathrm{EQCD}}$ is the perturbative $3 \mathrm{~d} \leftrightarrow 4 \mathrm{~d}$ matching coefficient for pressure. This is perturbatively computable order-by-order in coupling constant expansion, because all perturbatively problematic infrared singularities of high temperature QCD are fully contained in EQCD. The matching coefficient is currently known to order $\mathcal{O}\left(g^{5}\right)$ [17], 2

The simulation results in Sec. 3 indicate that the $\mathcal{O}\left(g^{6}\right)$ and higher order contributions to the matching coefficient are very small; indeed, if we compare our results with the $4 \mathrm{~d}$ simulation results, we obtain an excellent fit when we assume that these contributions vanish. Thus, the $\mathcal{O}\left(g^{6}\right)$ and above contributions to the susceptibility are strongly dominated by the contributions coming from EQCD.

Because EQCD is derived using perturbation theory, the final results depend on the perturbative scale $\Lambda_{\overline{\mathrm{MS}}}$. We shall use here the value $\Lambda_{\overline{\mathrm{MS}}}=245 \mathrm{MeV}$, which has been obtained from lattice simulations with 2 light Wilson quarks [30]. For the critical temperature we use $T_{\mathrm{c}}=170 \mathrm{MeV}$, yielding the ratio $T_{\mathrm{c}} / \Lambda_{\overline{\mathrm{MS}}}=0.73$ The comparison between EQCD and $4 \mathrm{~d}$ QCD simulation results is somewhat sensitive to the precise value of this ratio, but it can vary $\pm 10 \%$ without significantly affecting the quality of the match. The value 0.7 turns out to be close to the optimal one for the matching.

Due to the perturbative nature of the matching equations it turns out to be convenient to do the matching by subtracting the known $3 \mathrm{~d}$ perturbative susceptibility and adding the $4 \mathrm{~d}$ one:

$$
\frac{\chi}{T^{2}}=\frac{g_{3}^{6}}{\pi^{2} T^{3}}\left(\chi_{3}^{\text {latt }}-\chi_{3}^{\text {pert }}\right)+\frac{\chi^{\text {pert }}}{T^{2}} .
$$

Here $\chi_{3}^{\text {pert }}$ and $\chi^{\text {pert }}$ are $3 \mathrm{~d}$ and $4 \mathrm{~d}$ perturbative results. We also note that the quantities $\chi_{\mathrm{uu}}=\chi_{\mathrm{dd}}$ and $\chi_{\mathrm{ud}}=\chi_{\mathrm{du}}$ are related to those used in [5] by

$$
\begin{aligned}
\chi_{\mathrm{q}} & =2\left(\chi_{\mathrm{uu}}+\chi_{\mathrm{ud}}\right) \\
\chi_{\mathrm{I}} & =\frac{1}{2}\left(\chi_{\mathrm{uu}}-\chi_{\mathrm{ud}}\right) \\
\chi_{\mathrm{C}} & =\frac{5}{9} \chi_{\mathrm{uu}}-\frac{4}{9} \chi_{\mathrm{ud}} .
\end{aligned}
$$

\footnotetext{
${ }^{2}$ For the pressure the matching coefficient has been calculated to $\mathcal{O}\left(g^{6}\right)$ in a much simpler theory in Ref. 29.

${ }^{3}$ We obtain the same value by using the results $r_{0} T_{c}=0.438$ [31] and $r_{0} \Lambda_{\overline{\mathrm{MS}}}=0.62$ [30].
} 


\subsection{On the lattice}

The theory in Eq. (2.1) is discretized in a standard way, as described in [16]. Due to the superrenormalizability of the $3 \mathrm{~d}$ theory the couplings $\lambda_{3}$ and $g_{3}^{2}$ do not run, and $m_{3}^{2}$ has well-known linear and logarithmic divergences as the lattice spacing $a \rightarrow 0$. When these divergences are subtracted the continuum limit is straightforward.

The evaluation of the quark number susceptibility requires the measurement of the condensates in Eq. (2.13) on the lattice. Due to the superrenormalizable nature of the theory, measurements can be rigorously converted to $\overline{\mathrm{MS}}$ scheme in the lattice continuum limit; because $\overline{\mathrm{MS}}$ was used in in the perturbative matching to $4 \mathrm{~d} \mathrm{QCD}$, this also allows us to compare to $4 \mathrm{~d}$ results.

The relations between the condensates on the lattice and in continuum can be written in the limit the lattice spacing $a \rightarrow 0$ (or $\beta \equiv 6 /\left(g_{3}^{2} a\right) \rightarrow \infty$ ) as [16, 32]

$$
\begin{aligned}
& C_{1, \overline{\mathrm{MS}}}=C_{1, a}-\tilde{c}_{1} \beta-\tilde{c}_{2}\left(\ln \beta+{\tilde{c_{2}}}^{\prime}\right)+\mathcal{O}(1 / \beta), \\
& C_{2, \overline{\mathrm{MS}}}=C_{2, a}-\left[\bar{c}_{2}\left(\ln \beta+\bar{c}_{2}^{\prime}\right)\right]+\mathcal{O}(1 / \beta), \\
& C_{3, \overline{\mathrm{MS}}}=C_{3, a}+\mathcal{O}(1 / \beta), \\
& C_{4, \overline{\mathrm{MS}}}=C_{4, a}+\mathcal{O}(1 / \beta) .
\end{aligned}
$$

Here labels $\overline{\mathrm{MS}}$ and $a$ indicate that the quantity is calculated in $\overline{\mathrm{MS}}$ or lattice regularization, respectively. The numerical coefficients are

$$
\begin{aligned}
\tilde{c}_{1} & \approx 0.1684873399, \\
\tilde{c}_{2} & =\frac{3 d_{\mathrm{A}}}{(4 \pi)^{2}} \approx 0.1519817755, \\
\tilde{c}_{2}^{\prime} & \approx 0.66796(1), \\
\bar{c}_{2} & =\frac{5}{16 \pi^{2}} \approx 0.0316628698900405, \\
\bar{c}_{2}^{\prime} & \approx 0.08848010 .
\end{aligned}
$$

\section{Lattice simulations}

The lattice simulations were carried out using two massless quark flavors $\left(N_{\mathrm{f}}=2\right)$. We used nine different values of temperature $T$, varying from $T \approx 1.9 \Lambda_{\overline{\mathrm{MS}}}$ up to $\sim 9 \times 10^{16} \Lambda_{\overline{\mathrm{MS}}}$. The temperature values are shown in Table 3. While the largest temperature is huge in physical units, in $3 \mathrm{~d}$ parameters the variation is much milder; this is related to the fact that QCD approaches weakly coupled theory at high $T$ extremely slowly. Thus, an extreme range of high temperatures is required in order to reliably assess the convergence to the perturbation theory.

At each temperature we use 6 values for $z=\left(\mu_{\mathrm{u}}+\mu_{\mathrm{d}}\right) /\left(3 \pi^{2} T\right), \mu_{\mathrm{u}}=\mu_{\mathrm{d}}$, up to $z=0.15$ or $\mu_{\mathrm{u}} / T \approx 2.22$. This amounts to 54 different $(T, \mu)$ pairs. For each physical point simulations 


\begin{tabular}{|l|l|l|}
\hline$T / T_{\mathrm{c}}$ & $y_{0}(T)$ & $x(T)$ \\
\hline 1.32 & 0.357 & 0.13 \\
2.31 & 0.448 & 0.10 \\
11.5 & 0.711 & 0.06 \\
204 & 1.18 & 0.035 \\
3600 & 2.02 & 0.020 \\
$2.4 \times 10^{7}$ & 3.09 & 0.013 \\
$6.2 \times 10^{9}$ & 3.99 & 0.010 \\
$1.9 \times 10^{13}$ & 5.31 & 0.0075 \\
$6.1 \times 10^{16}$ & 6.62 & 0.006 \\
\hline
\end{tabular}

Table 1: The temperatures and corresponding $y_{0}, x$-values used in the simulations. For each temperature quark chemical potential has 6 values, parametrized by $z \equiv 2 \mu_{q} /\left(3 \pi^{2} T\right)=$ $0,0.025,0.05,0.075,0.1,0.15$, and for non-zero $z y$ is modified according to Eq. (2.9). At each $(T, \mu)$-pair the simulations are done using 6 different lattice spacings, parametrized by $\beta \equiv 6 /\left(g_{3}^{2} a\right)=32,40,54,67,80,120$.

are done using 6 lattice spacings, parametrized by $\beta=6 /\left(g_{3}^{2} a\right)=32 \ldots 120$. Thus, the lattice spacings vary by almost a factor of 4 , enabling reliable extrapolation of the continuum limit. For the smallest lattice spacing $(\beta=120)$, the largest lattice size varies between $256^{3}$ to $320^{3}$.

In addition to the simulations at physical parameter values, we also did a several series of runs at fixed $x, y$ and varying $z$. While these simulations do not correspond to any physical parameter set, they enable us to look at the $z$-dependence of the condensates separately. All in all, our dataset contains 693 individual runs.

\subsection{Continuum extrapolation}

It turns out that the accuracy requirement are so high that the continuum limit extrapolation of the condensates have to be taken with great care. Especially the continuum extrapolation of $\left\langle\hat{A}_{0}^{2}\right\rangle$ is critical, because it strongly dominates the susceptibility. While we know the divergent (as $a \rightarrow 0$ ) and constant contributions appearing in the continuum limit, Eq. (2.22), $\mathcal{O}(a)$, $\mathcal{O}(a \ln a)$ and higher order terms are not yet known. Thus, we use an ansatz

$$
\left\langle\hat{A}_{0}^{2}\right\rangle_{a}-C . T .=c_{1}+\frac{c_{2}}{\beta}+\frac{c_{2}^{\prime}}{\beta} \log (\beta)+\frac{c_{3}}{\beta^{2}},
$$

where C.T. indicate the known counterterms in Eq. (2.22) and $c_{i}$ are fit parameters. The existence of the logarithmic term in the ansatz increases the errors of the extrapolation an order of magnitude compared to the case without the logarithmic term. However, $c_{2}^{\prime}$ is expected to be a constant independent of $y$ : by dimensional grounds the expansion of $\left\langle\hat{A}_{0}^{2}\right\rangle$ 
in powers of the lattice spacing can be written as

$$
\left\langle\hat{A}_{0}^{2}\right\rangle_{a}=\frac{D_{1}}{a}+D_{2} g_{3}^{2}+a\left[D_{3} g_{3}^{4}+D_{4} m_{3}^{2}+D_{5} g_{3}^{2} \lambda+D_{6} \lambda^{2}\right]+\mathcal{O}\left(a^{2}\right) .
$$

The form of the $\mathcal{O}(a)$ coefficients $D_{4}, D_{5}$ and $D_{6}$ is known and they do not contain a term logarithmic in $a$ [16, 32, whereas the coefficient $D_{3}$, which is constant in $y$ (or $m_{3}^{2}$ ), might include one. Thus, the possible $a \ln a$-contribution should indeed be independent of $y$.

The existence of the logarithmic term can be seen in Fig. 1, where we show the parameter $c_{2}^{\prime}$ obtained from continuum fits using the ansatz (3.1). Note that here $c_{2}^{\prime}$ is fitted independently for each physical parameter set, allowing arbitrary $y$ (and $z$ ) dependence. As expected, the result is fairly well consistent with constant $c_{2}^{\prime} \approx 0.69$; the remaining systematic discrepancies in the fit can be caused by contributions which are of higher order than $\mathcal{O}\left(a^{2}\right)$, including terms of type $a^{2} \ln a$. Thus, we shall fix $c_{2}^{\prime}$ to this value in Eq. (3.1) for all continuum limit extrapolations which follow.

We note that the value of $c_{2}^{\prime}$ has negligible effect on the results at small (physically relevant) temperatures; $c_{2}^{\prime}$ could be set to zero without affecting the continuum limit. It is significant only at very large $T$, where it potentially has a role when we compare simulations with the perturbation theory. We observe deviations from perturbative results even at very high $T$ if $c_{2}^{\prime} \lesssim 0.4$ (see also [26]). However, variations of order $\sim 15 \%$ around 0.69 do not affect the final results.

Nevertheless, it is clear that an analytic calculation of $\mathcal{O}(a)$ effects in EQCD would be highly desirable. There is an ongoing calculation using stochastic perturbation theory [33], which will hopefully confirm our results.

The contributions of the other condensates are numerically much smaller and we were not able to see any sign of logarithmic $a$-dependence in those. It turns out that it is advantageous to make the continuum extrapolation using the full expression of the susceptibility (2.16), instead of extrapolating individual condensates. (Naturally, after the subtraction of the known counterterms in Eq. (2.22).) This extrapolation is shown in Fig. 2 ,

The lattice volumes are chosen large enough so that finite volume effects become negligible. We have tested this by doing simulations at selected parameter values using different volumes; at the smallest lattice spacing $(\beta=120)$ the volume varies from $V=144^{3}$ up to $V=320^{3}$. No systematic finite volume effects inside two sigma errors. For more discussion of finite size effects on a related model see Ref. [21].

The finite chemical potential dependence is studied using the method described in Sec. 2.3. The condensates $C_{2}=V g_{3}^{6}\left(\left\langle\left(\hat{A}_{0}^{3}\right)^{2}\right\rangle-\left\langle\hat{A}_{0}^{3}\right\rangle^{2}\right)$ and $C_{3}=V g_{3}^{6}\left(\left\langle\left(\hat{A}_{0}^{2}\right)^{2}\right\rangle-\left\langle\hat{A}_{0}^{2}\right\rangle^{2}\right)$ should be largely independent of $z$ (for fixed $x, y$ ) for the equation (2.15) to be valid. This indeed turns out to be the case, within the statistical errors, and any remnant $z$-dependence is completely drowned out by the contributions from $z$-independent parts in Eq. (2.15). Indeed, the overall $z$-dependence of each of the condensates in Eq. (2.15) turns out to be statistically almost invisible, with the exception of $C_{4}=V g_{3}^{2}\left(\left\langle\hat{A}_{0}^{2} \hat{A}_{0}^{3}\right\rangle-\left\langle\hat{A}_{0}^{2}\right\rangle\left\langle\hat{A}_{0}^{3}\right\rangle\right)$, which has a linear 


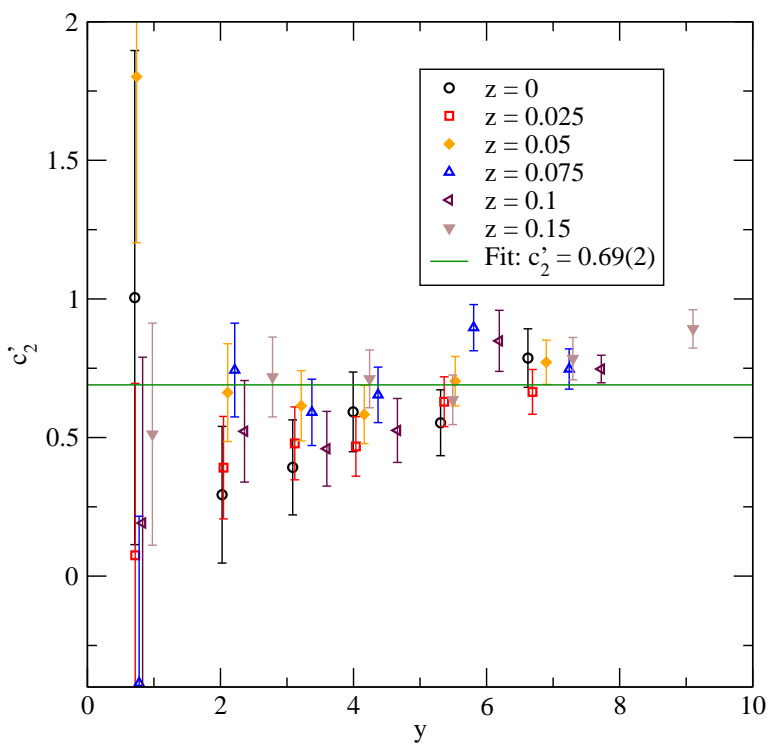

Figure 1: Fitting of the logarithmic coefficient $c_{2}^{\prime}$ in continuum extrapolation. The data is consistent with the assumption that $c_{2}^{\prime}$ is a constant $\chi^{2} /$ d.o.f $\approx 57 / 35$
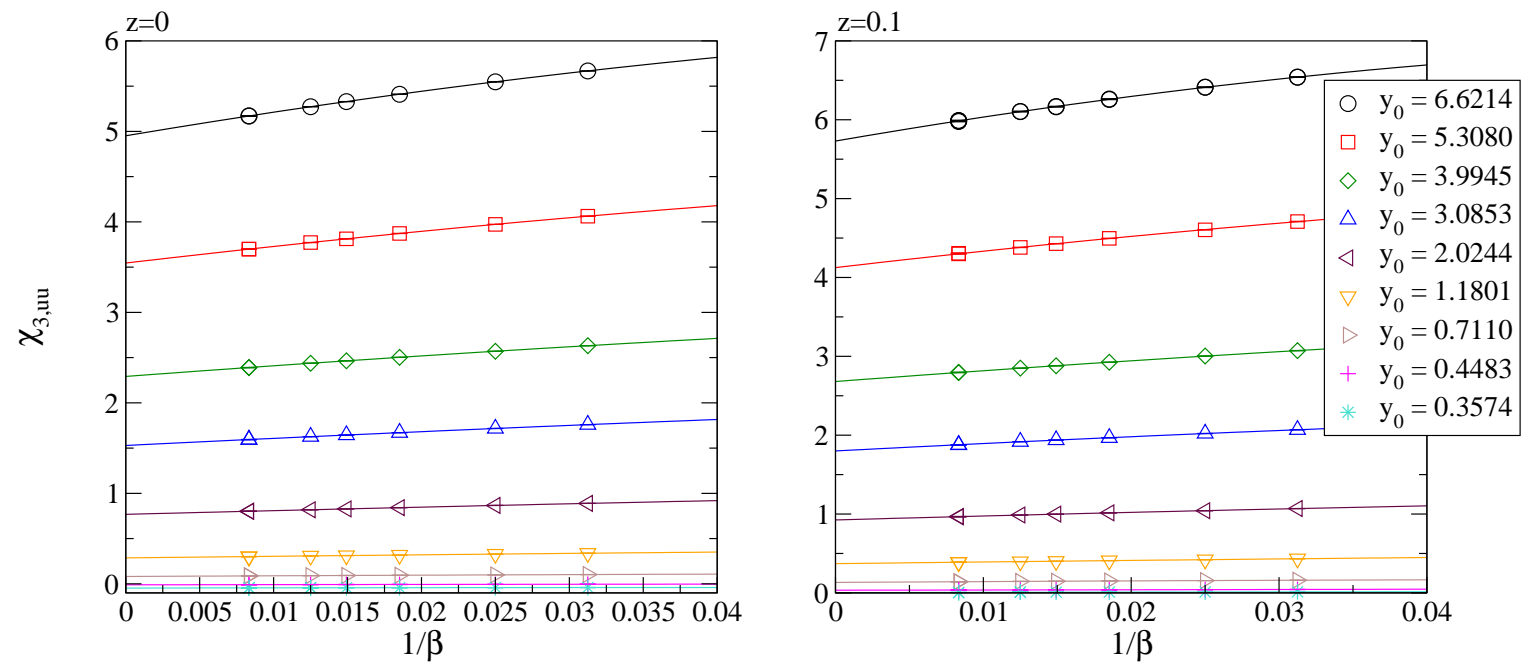

Figure 2: Continuum extrapolation of the diagonal susceptibility $\chi_{3, \text { uu }}$ at chemical potential $z=0$ and $z=0.1$. The statistical errors are too small to be visible. 
$z$-dependence. In practice the $\mu$-dependence of the susceptibility is almost completely due to the $\mu$-dependence of the parameter $y$ and the $\mu^{2} C_{3}$-term Eq. (2.16) 4 . Nevertheless, here we do take into account the small $z$-dependence of $C_{1}=\left\langle\hat{A}_{0}^{2}\right\rangle$ and $C_{4}$, although it will affect the final results by only about 1 sigma.

\subsection{Diagonal susceptibility}

Now we are in position to compare the continuum limit results with the perturbation theory. First we shall look at the diagonal susceptibility $\chi_{3 \text {,uu }}=\chi_{3 \text {,dd }}$. The susceptibility has been calculated in perturbation theory up to order $g^{6} \ln 1 / g$ [9]. In 3-dimensional units the perturbative result can be written as a power series in $1 / \sqrt{y_{0}}$, with the following result:

$$
\begin{aligned}
\frac{\chi_{3, \text { uu }}^{\text {pert }}}{g_{3}^{6}}= & \frac{8+9 \bar{\mu}^{2}}{4 \sqrt{4+3 \bar{\mu}^{2}}} \frac{3 y_{0}^{3 / 2}}{4 \pi} \\
- & \frac{(9-30 x) \bar{\mu}^{2}-4(3+10 x)+6\left(4+3 \bar{\mu}^{2}\right) \ln \left(4+3 \bar{\mu}^{2}\right)+6\left(4+3 \bar{\mu}^{2}\right) \ln \left(y_{0}\right)}{2\left(4+3 \bar{\mu}^{2}\right)} \frac{3 y_{0}}{(4 \pi)^{2}} \\
- & \frac{\left(8+3 \bar{\mu}^{2}\right)\left(89+4 \pi^{2}-44 \ln (2)\right)}{8\left(4+3 \bar{\mu}^{2}\right)^{3 / 2}} \frac{9 y_{0}^{1 / 2}}{(4 \pi)^{3}} \\
+ & \left\{576\left[-3438+40\left(2+\bar{\mu}^{2}\right) \bar{\mu}^{2}\right]+119313 \pi^{2}+640\left(4+3 \bar{\mu}^{2}\right)^{2} \ln \left(4+3 \bar{\mu}^{2}\right)\right. \\
& \left.\quad+640\left(4+3 \bar{\mu}^{2}\right)^{2} \ln \left(y_{0}\right)\right\} \frac{1}{144\left(4+3 \bar{\mu}^{2}\right)^{2}(4 \pi)^{4}}+\frac{80}{3(4 \pi)^{4}} \beta_{\mathrm{M} 2}+\mathcal{O}\left(y_{0}^{-1 / 2}\right) .
\end{aligned}
$$

We have set here $\bar{\mu}_{\mathrm{u}}=\bar{\mu}_{\mathrm{d}}=\bar{\mu}$. As can be observed in Fig. 3 the overall agreement between the lattice result and the perturbation theory is very good, especially at large $y$ (large temperature). The result contains an unknown $\bar{\mu}$-independent order $\mathcal{O}\left(y_{0}^{0}\right)$-term denoted by $\beta_{\mathrm{M} 2}$ in [18]. The same term appears also in the off-diagonal susceptibility, Eq. (3.5), and it turns out that it gives much tighter constraints for the value of $\beta_{\mathrm{M} 2}$ than the diagonal one. The fit to the off-diagonal susceptibility gives $\beta_{\mathrm{M} 2}=-0.1 \pm 0.3$. This value is small enough that its effect is negligible for the diagonal susceptibility, nonetheless we set here $\beta_{\mathrm{M} 2}=-0.1$.

In order to quantify the contributions not included in the perturbative result we calculate the difference $\chi_{3}^{\text {latt }}-\chi_{3}^{\text {pert }}$ and fit a function of form $b_{1} / y_{0}^{-1 / 2}+b_{2} / y_{0}$ to the result. The fit results are shown in Fig. 4 and Table 2. We note that for small $z$ the $1 / y_{0}^{1 / 2}$-term is much smaller than the $1 / y_{0}$-term, indicating that the $\mathcal{O}\left(g^{7}\right)$-contribution arising from EQCD is smaller in magnitude to the $\mathcal{O}\left(g^{8}\right)$ term, at least for all physically relevant temperatures. At large $z$ the statistical errors grow rapidly; this is due to the term $\propto z^{2} V g_{3}^{6}\left(\left\langle\left(\hat{A}_{0}^{2}\right)^{2}\right\rangle-\left\langle\hat{A}_{0}^{2}\right\rangle^{2}\right)$ in Eq. (2.16).

Finally, we obtain the physical $4 \mathrm{~d}$ result for the diagonal susceptibility from Eq. (2.18). As described in Sec. 2.4, the $3 \mathrm{~d} \leftrightarrow 4 \mathrm{~d}$ mapping remains sensitive to the unknown $\mathcal{O}\left(g^{6}\right)$ and higher order perturbative contributions to the matching coefficient. In Fig. 5 we show the

\footnotetext{
${ }^{4}$ This fact was used in the preliminary results published in ref. [27]
} 

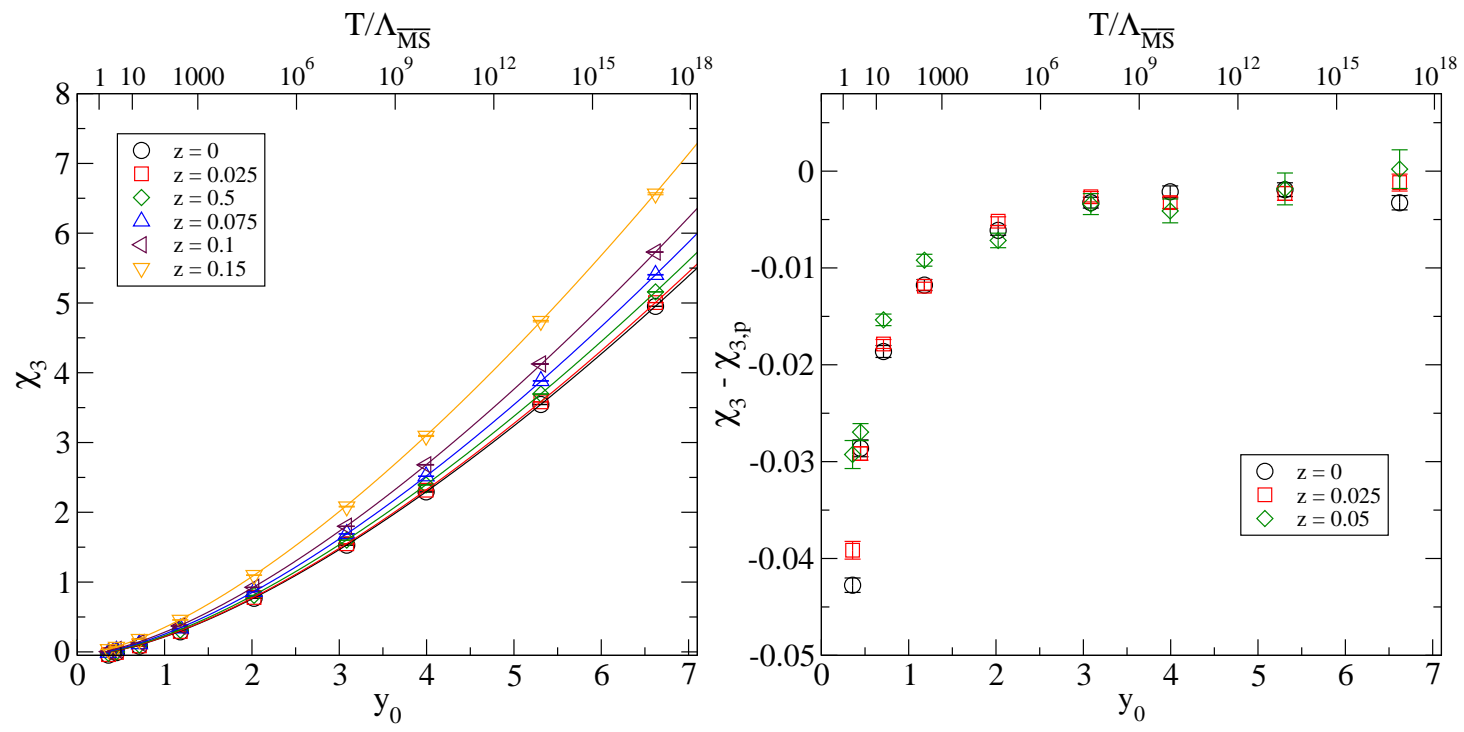

Figure 3: Left: the diagonal quark number susceptibility $\chi_{3, u u} / g_{3}^{6}$ at different values of chemical potential. The symbols indicate the lattice measurements, and the solid lines are the perturbative result. Right: The difference between the lattice and perturbation theory.

\begin{tabular}{|c|c|c|}
\hline $\mathrm{z}$ & fit & $\chi^{2} /$ dof \\
\hline 0 & $0.0008(8) / \sqrt{y_{0}}-0.0137(8) / y_{0}$ & $12 / 6$ \\
0.025 & $0.0016(8) / \sqrt{y_{0}}-0.0143(7) / y_{0}$ & $18 / 6$ \\
0.05 & $0.000(1) / \sqrt{y_{0}}-0.012(2) / y_{0}$ & $11 / 6$ \\
0.075 & $-0.003(2) / \sqrt{y_{0}}-0.007(2) / y_{0}$ & $17 / 6$ \\
0.1 & $-0.008(4) / \sqrt{y_{0}}-0.002(3) / y_{0}$ & $7.5 / 6$ \\
0.15 & $-0.012(6) / \sqrt{y_{0}}+0.004(5) / y_{0}$ & $9.7 / 6$ \\
\hline
\end{tabular}

Table 2: Fitting a function of form $b_{1} / \sqrt{y_{0}}+b_{2} / y_{0}$ to $\left(\chi_{3, \mathrm{uu}}^{\text {lat }}-\chi_{3, \mathrm{uu}}^{\text {pert }}\right) / g_{3}^{6}$. The smallest $y$ (lowest temperature) points are left out of the fit. 

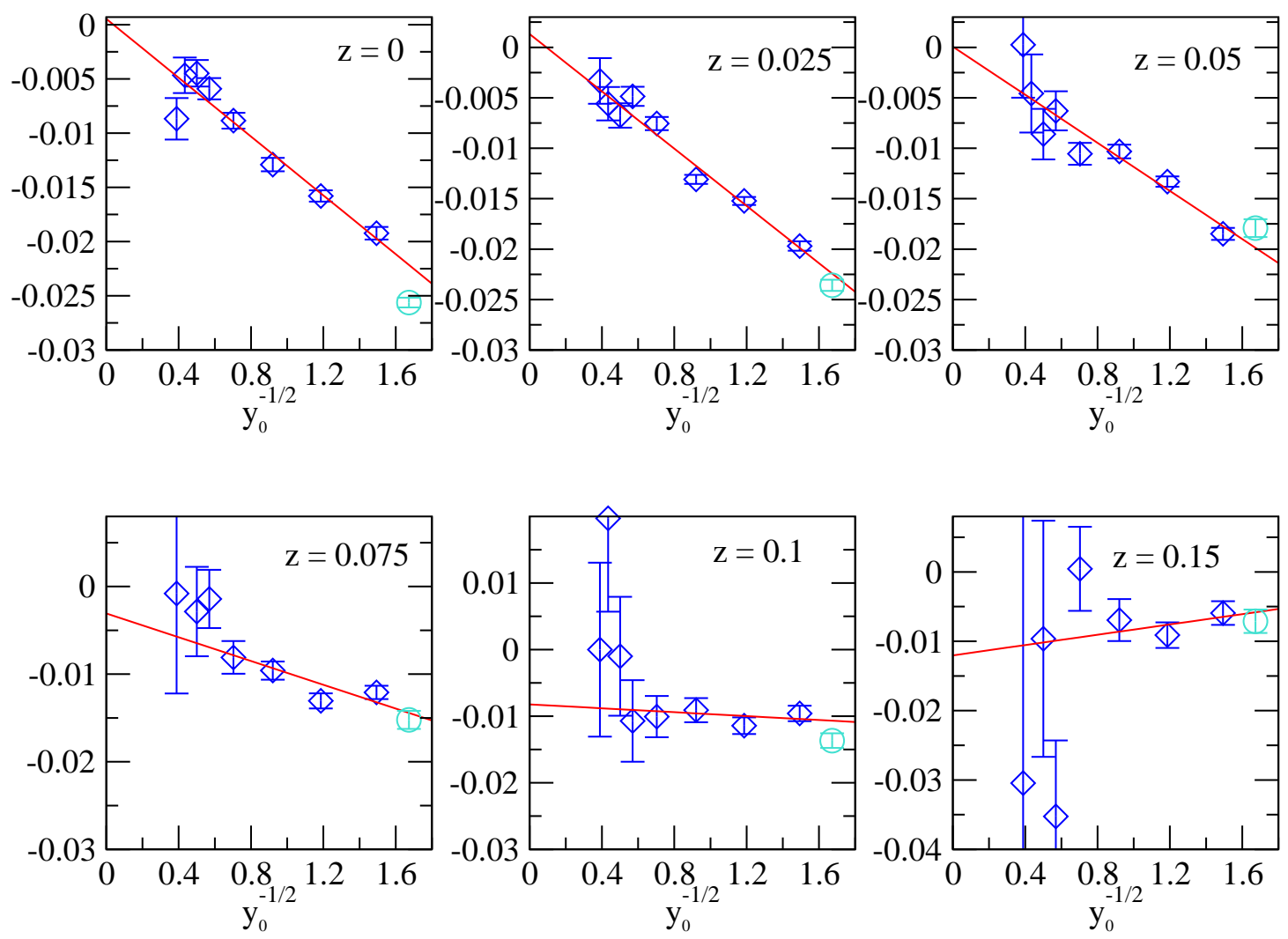

Figure 4: The diagonal susceptibility $\left(\chi_{3, \mathrm{uu}}^{\text {lat }}-\chi_{3, \mathrm{uu}}^{\text {pert }}\right) \sqrt{y} / g_{3}^{6}$ as a function of $1 / \sqrt{y}$ with different values of the chemical potential. Solid line is a 1 st order polynomial fit. The data at $y_{0}^{-1 / 2} \approx$ 1.6 have been excluded from the fit.

EQCD data at $\bar{\mu}=0$ with these unknown contributions set to zero. We observe that the result fits the $4 \mathrm{~d}$ lattice simulations very well, clearly indicating that the magnitude of these contributions must be small, and in what follows we shall set them to zero. On the other hand, it should be noted that the difference between the purely perturbative result and EQCD simulation result is substantial at $T \lesssim 10 T_{\mathrm{c}}$, as indicated by the two lines in Fig. 5. This is a clear indication that the contributions beyond the currently known perturbative ones have non-negligible effect at experimentally accessible temperatures.

The $\mu$-dependence of the diagonal susceptibility is shown in Fig. 6, normalized to the Stefan-Boltzmann value

$$
\chi_{\mathrm{SB}}(\mu)=T^{2}+\frac{3}{\pi^{2}} \mu^{2} .
$$

We note that at temperatures above $100 T_{\mathrm{c}}$ the deviation from the Stefan-Boltzmann law is independent of $\mu$, but at lower $T$ there is significant $\mu$-dependence. The $\mu$-dependence matches very well the $4 \mathrm{~d}$ lattice results by Allton et al. [5], also shown in Fig. 6. 


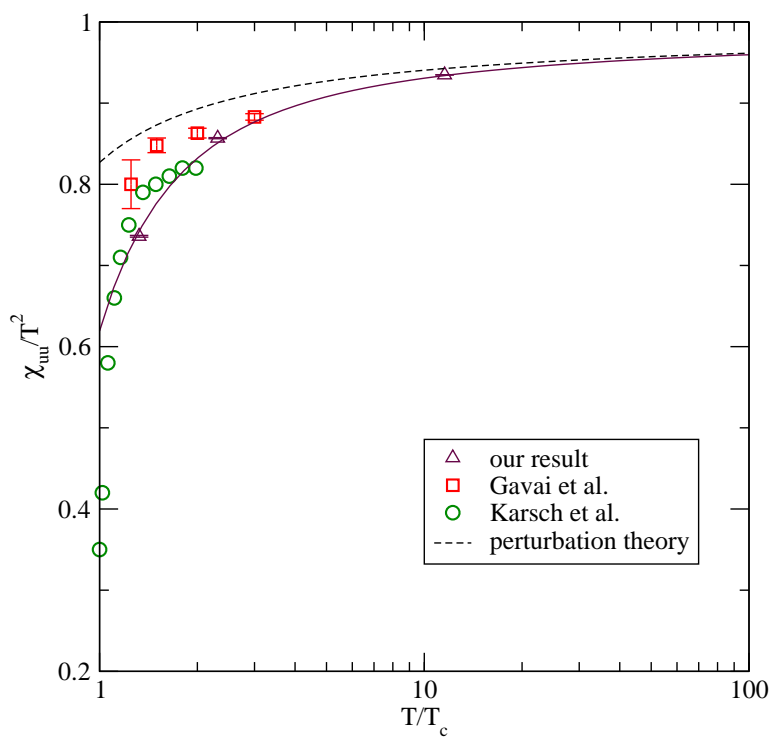

Figure 5: The diagonal susceptibility $\chi_{\mathrm{uu}}$ in $4 \mathrm{~d}$ units at $\mu=0$. The data points indicate the full EQCD result. The continuous line is the result of the fit in Table 2 , The dashed line shows the perturbative result alone, Eq. (3.3), using the same matching as in the EQCD result. The difference between these two curves indicates the magnitude of the non-perturbative contributions. The agreement with the 4d-lattice results of Gavai et al. [2] and Karsch et al. [4] is good.
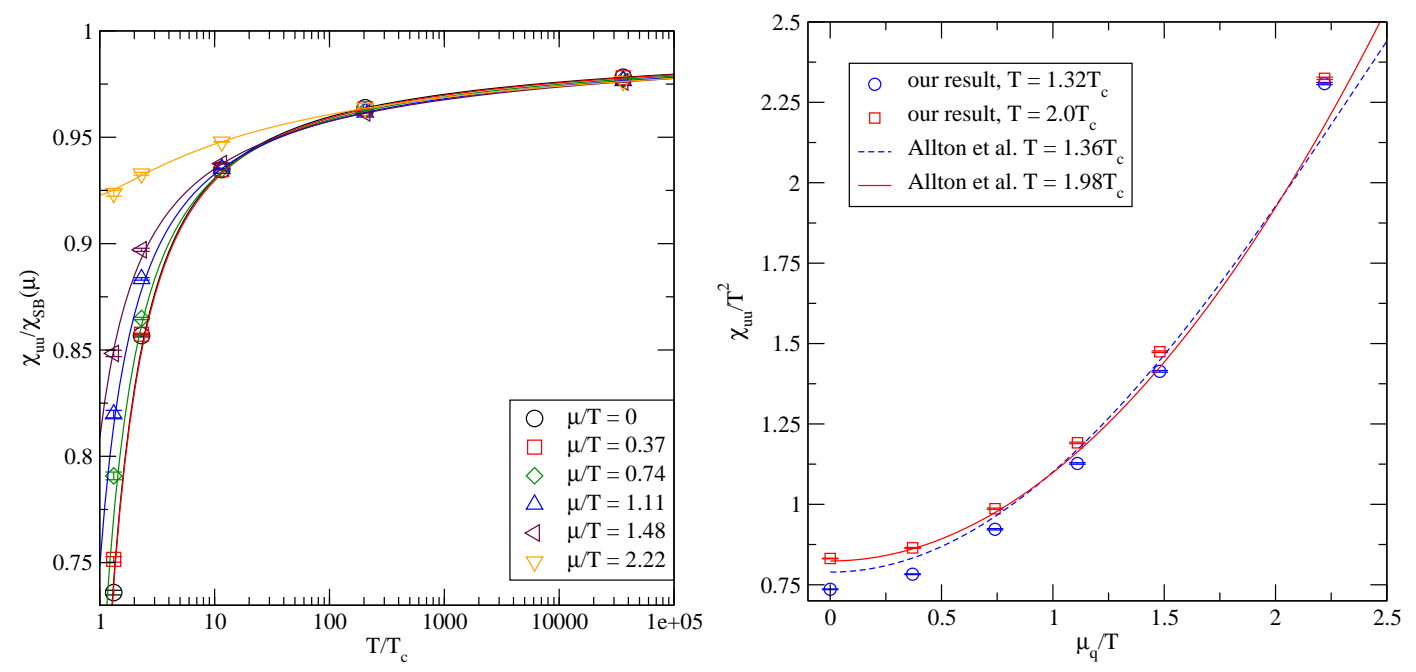

Figure 6: Left: The diagonal susceptibility at different $\mu$, normalized to Stefan-Boltzmann law. Right: $\mu$-dependence of the susceptibility compared with the $4 \mathrm{~d}$ lattice results of Allton et al. [5]. 


\subsection{Off-diagonal susceptibility}

The perturbative result for the off-diagonal susceptibility in $3 \mathrm{~d}$ units is

$$
\begin{aligned}
\frac{\chi_{3, \mathrm{ud}}^{\text {pert }}}{g_{3}^{2}}= & \frac{9 \bar{\mu}^{2}}{4 \sqrt{4+3 \bar{\mu}^{2}}} \frac{y_{0}^{3 / 2}}{4 \pi} \\
+ & \frac{27 \bar{\mu}^{2}}{4+3 \bar{\mu}^{2}} \frac{y_{0}}{(4 \pi)^{2}} \\
+ & \frac{27 \bar{\mu}^{2}\left(89+4 \pi^{2}-44 \log (2)\right)}{8\left(4+3 \bar{\mu}^{2}\right)^{3 / 2}} \frac{y_{0}^{1 / 2}}{(4 \pi)^{3}} \\
+ & \left\{3\left(2047168-119313 \pi^{2}+15360 \bar{\mu}^{2}\right) \bar{\mu}^{2}+2560\left(4+3 \bar{\mu}^{2}\right)^{2} \ln \left(4+3 \bar{\mu}^{2}\right)+\right. \\
& \left.2560\left(4+3 \bar{\mu}^{2}\right) \ln \left(y_{0}\right)\right\} \frac{1}{576\left(4+3 \bar{\mu}^{2}\right)^{2}(4 \pi)^{4}}+\frac{80}{3(4 \pi)^{4}} \beta_{\mathrm{M} 2}+\mathcal{O}\left(y_{0}^{-1 / 2}\right)
\end{aligned}
$$

where $\beta_{\mathrm{M} 2}$ is the same unknown coefficient which appears in the diagonal susceptibility, Eq. (3.3). In this case we can fit the value at $z=0$, obtaining

$$
\beta_{\mathrm{M} 2}=-0.1 \pm 0.3
$$

This value is small enough to have in practice negligible effect on the final results. Again the simulation data is very well described by the perturbation theory, Fig. 7, only at $z=0$ or at lowest temperatures can we observe deviations from perturbation theory.

After matching to $4 \mathrm{~d}$, we obtain the result for off-diagonal susceptibility $\chi_{\mathrm{ud}}$, shown in Fig. 8. At $T \gtrsim 10 T_{\mathrm{c}}$ the results match the perturbation theory very well, but at lower temperatures there are deviations: most significantly, at $T=1.32 T_{\mathrm{c}}$ and $\mu=0$ the simulation results clearly undershoot the perturbation theory. On the other hand, the $4 \mathrm{~d}$ lattice results in [5] at $\mu=0$ indicate small but non-zero value, which agrees well with perturbation theory. [9, 10]. This can be an indication that this point is already outside the validity range of EQCD; however, we also note that by increasing $T_{\mathrm{c}} / \Lambda_{\overline{\mathrm{MS}}}$ the EQCD results are brought closer to $4 \mathrm{~d}$ lattice results [5]. The agreement with the perturbation theory and $4 \mathrm{~d}$ lattice results is rather good already at $T=2.3 T_{\mathrm{c}}$.

We also note that the physical value of the off-diagonal susceptibility is obtained in EQCD by a subtraction of two divergent as $a \rightarrow 0$ terms; thus, as opposed to full 4d QCD simulation, there is no natural approximate symmetry which would force it to be small. Therefore, if EQCD starts to approach the limits of the validity, one can expect substantial deviations from physical results, as seen at $T=1.32 T_{\mathrm{c}}$.

Nevertheless, the overall $\mu$-dependence of $\chi_{\mathrm{ud}}$ is in rough accordance with the $4 \mathrm{~d}$ lattice results [5] already at $T=1.32 T_{\mathrm{c}}$, as shown on the right panel in Fig. 8 , and at $T=2.3 T_{\mathrm{c}}$ the agreement is already very good. The non-diagonal susceptibility is seen to behave quite well up to large values of $\mu / T \sim 2$. 

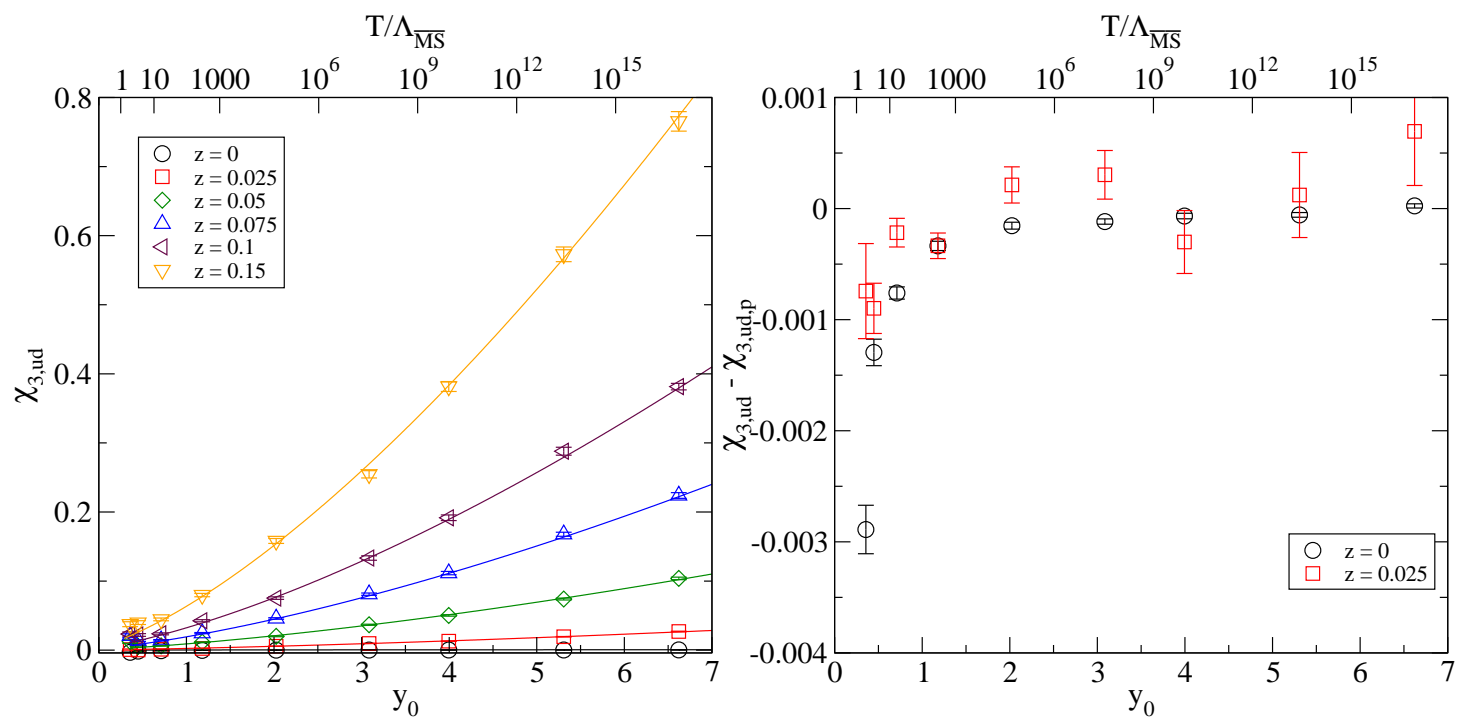

Figure 7: Left: the off-diagonal susceptibility $\chi_{3, \mathrm{ud}} / g_{3}^{6}$ in $3 \mathrm{~d}$ units. Right: the difference between lattice and perturbative susceptibilities $\left(\chi_{3, \mathrm{ud}}^{\text {latt }}-\chi_{3, \mathrm{ud}}^{\text {pert }}\right) / g_{3}^{6}$, shown at 2 smallest $\bar{\mu}$. The statistical errors grow rapidly as $\bar{\mu}$ increases.
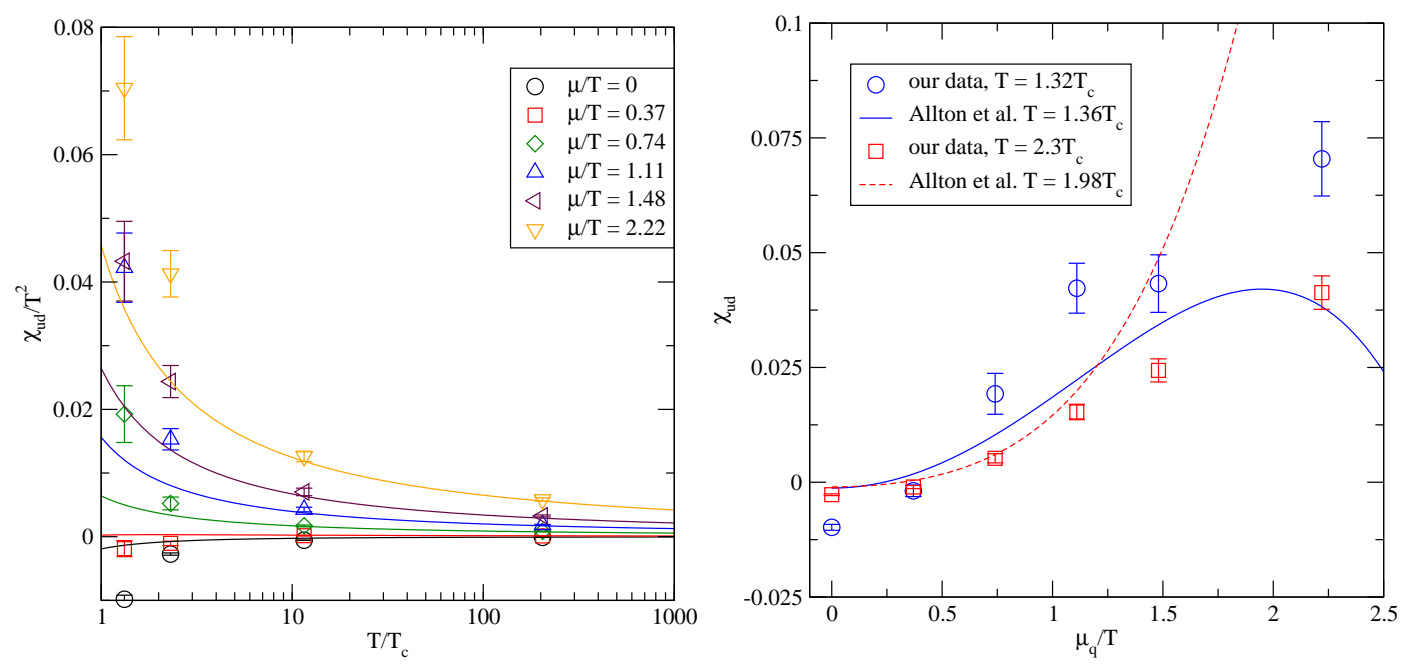

Figure 8: Left: The off-diagonal susceptibility in 4d. At low temperatures we obtain significantly different values from the perturbation theory (solid lines), but there is no deviation anymore at $T=10 T_{\mathrm{c}}$. Right: $\mu$-dependence of off-diagonal susceptibility compared with Allton et al. [5]. The precision of results from [5] are probably not accurate enough to predict the behaviour at region $\mu / T>1$. 


\section{Conclusions}

We have measured the quark number susceptibility of high temperature finite density QCD using lattice simulations of EQCD, an effective 3-dimensional theory of full 4d QCD. The very good match to the $4 \mathrm{~d}$ lattice results with 2 light quark flavors at low temperatures and with the perturbation theory at high temperatures shows the wide range of applicability of the method. The diagonal susceptibility is seen to agree with $4 \mathrm{~d}$ simulations by Allton et al. 5] even below $2 T_{\mathrm{c}}$, including the dependence on $\mu$. On the other hand, we observe a substantial deviation from the known perturbative result up to temperatures $\sim 20 T_{\mathrm{c}}$. The off-diagonal susceptibility is compatible with perturbation theory already at $T \gtrsim 10 T_{\mathrm{c}}$. The results also agree with the $4 \mathrm{~d}$ simulations [5] except perhaps at lowest temperatures, $T<2 T_{\mathrm{c}}$.

The results clearly indicate that EQCD is a viable method to obtain quantitatively significant results of the hot QCD plasma down to $T \sim 2 T_{\mathrm{c}}$. Equally significant is the observation that the currently known perturbative result alone deviates significantly from the correct result: while the perturbative result can be made to match the $4 \mathrm{~d}$ lattice data by adjusting the still unknown (high perturbative order) matching coefficients, EQCD allows us to directly measure the differences between simulations and perturbative calculations without any scale or matching ambiguities. Thus, simulations of EQCD are exceptionally well suited for observing the convergence of the perturbation theory. It is worth noting that while the EQCD susceptibility also suffers from matching ambiguity, we obtain an excellent fit to $4 \mathrm{~d}$ simulations by assuming these matching coefficients vanish, indicating that the contribution from these is necessarily very small.

\section{Acknowledgements}

We acknowledge useful discussion with K. Kajantie, M. Laine and A. Vuorinen. This work has been partly supported by the Magnus Ehrnrooth Foundation, a Marie Curie Fellowship for Early Stage Researchers Training, and the Academy of Finland, contract number 114371. KR also acknowledges partial support by the National Science Foundation under Grant No. PHY05-51164. Simulations have been carried out at the Finnish IT Center for Science (CSC).

\section{References}

[1] M. Asakawa, U. W. Heinz and B. Muller, Fluctuation probes of quark deconfinement, Phys. Rev. Lett. 85 (2000) 2072 arXiv:hep-ph/0003169;

S. Jeon and V. Koch, Charged particle ratio fluctuation as a signal for QGP, Phys. Rev. Lett. 85, 2076 (2000) arXiv:hep-ph/0003168.

[2] R. V. Gavai, S. Gupta and P. Majumdar, Susceptibilities and screening masses in two flavor QCD, Phys. Rev. D 65 (2002) 054506 hep-lat/0110032. 
[3] R. V. Gavai and S. Gupta, The continuum limit of quark number susceptibilities, Phys. Rev. D 65, 094515 (2002) arXiv:hep-lat/0202006.

[4] F. Karsch, S. Ejiri and K. Redlich, Hadronic fluctuations in the QGP, Nucl. Physifcon. A 774 (2006) 619 hep-ph/0510126].

[5] C. R. Allton, S. Ejiri, S. J. Hands, O. Kaczmarek, F. Karsch, E. Laermann and C. Schmidt, The equation of state for two flavor QCD at non-zero chemical potential, Phys. Rev. D 68 (2003) 014507 arXiv:hep-lat/0305007.

C. R. Allton et al., Thermodynamics of two flavor QCD to sixth order in quark chemical potential, Phys. Rev. D 71 (2005) 054508 arXiv:hep-lat/0501030.

[6] C. Bernard et al., QCD thermodynamics with $2+1$ flavors at nonzero chemical potential, Phys. Rev. D 77 (2008) 014503 [arXiv:0710.1330 [hep-lat]].

C. Bernard et al. [MILC Collaboration], QCD thermodynamics with three flavors of improved staggered quarks, Phys. Rev. D 71 (2005) 034504 arXiv:hep-lat/0405029.

[7] Y. Maezawa et al., Thermodynamics and heavy-quark free energies at finite temperature and density with two flavors of improved Wilson quarks, PoS LAT2007, 207 (2007) arXiv:0710.0945 [hep-lat]].

[8] M. Bluhm and B. Kampfer, Flavor Diagonal and Off-Diagonal Susceptibilities in a Quasiparticle Model of the Quark-Gluon Plasma, arXiv:0801.4147 [hep-ph].

[9] A. Vuorinen, Quark number susceptibilities of hot QCD up to $g^{* *} 6 \ln (g)$, Phys. Rev. D 67 (2003) 074032 hep-ph/0212283.

[10] J. P. Blaizot, E. Iancu and A. Rebhan, Quark number susceptibilities from HTLresummed thermodynamics, Phys. Lett. B 523, 143 (2001) arXiv:hep-ph/0110369.

[11] A. Ipp, K. Kajantie, A. Rebhan and A. Vuorinen, The pressure of deconfined QCD for all temperatures and quark chemical potentials, Phys. Rev. D 74, 045016 (2006) arXiv:hep-ph/0604060].

[12] P. H. Ginsparg, First Order And Second Order Phase Transitions In Gauge Theories At Finite Temperature, Nucl. Phys. B 170 (1980) 388.

[13] T. Appelquist and R. D. Pisarski, High-Temperature Yang-Mills Theories And ThreeDimensional Quantum Chromodynamics, Phys. Rev. D 23 (1981) 2305.

[14] K. Kajantie, M. Laine, K. Rummukainen and M. E. Shaposhnikov, Generic rules for high temperature dimensional reduction and their application to the standard model, Nucl. Phys. B 458 (1996) 90 hep-ph/9508379.

[15] E. Braaten and A. Nieto, Free Energy of QCD at High Temperature, Phys. Rev. D 53 (1996) 3421 hep-ph/9510408.

[16] K. Kajantie, M. Laine, K. Rummukainen and M. E. Shaposhnikov, 3d SU(N) + adjoint Higgs theory and finite-temperature QCD, Nucl. Phys. B 503 (1997) 357 arXiv:hep-ph/9704416. 
[17] K. Kajantie, M. Laine, K. Rummukainen and Y. Schröder, The pressure of hot QCD up to $g^{6} \ln (1 / g)$, Phys. Rev. D 67 (2003) 105008 hep-ph/0211321.

[18] A. Vuorinen, The pressure of QCD at finite temperatures and chemical potentials, Phys. Rev. D 68 (2003) 054017 hep-ph/0305183]. A. Vuorinen, The pressure of QCD at finite temperature and quark number density, arXiv:hep-ph/0402242.

[19] A. Gynther and M. Vepsalainen, Pressure of the standard model at high temperatures, JHEP 0601, 060 (2006) hep-ph/0510375.

[20] K. Kajantie, M. Laine, K. Rummukainen and Y. Schroder, How to resum longdistance contributions to the QCD pressure? Phys. Rev. Lett. 86 (2001) 10 arXiv:hep-ph/0007109].

[21] A. Hietanen, K. Kajantie, M. Laine, K. Rummukainen and Y. Schroder, Plaquette expectation value and gluon condensate in three dimensions, JHEP 0501 (2005) 013 hep-lat/0412008.

A. Hietanen and A. Kurkela, Plaquette expectation value and lattice free energy of threedimensional $S U(N(c)$ gauge theory, hep-lat/0609015.

[22] M. Laine and Y. Schroder, Two-loop QCD gauge coupling at high temperatures, JHEP 0503 (2005) 067 arXiv:hep-ph/0503061.

[23] K. Kajantie, M. Laine, J. Peisa, A. Rajantie, K. Rummukainen and M. E. Shaposhnikov, Non-perturbative Debye mass in finite T QCD, Phys. Rev. Lett. 79 (1997) 3130 arXiv:hep-ph/9708207.

[24] A. Hart, M. Laine and O. Philipsen, Static correlation lengths in QCD at high temperatures and finite densities, Nucl. Phys. B 586 (2000) 443 [hep-ph/0004060.

[25] A. Hart, M. Laine and O. Philipsen, Testing imaginary vs. real chemical potential in finite-temperature QCD, Phys. Lett. B 505, 141 (2001) arXiv:hep-lat/0010008].

[26] A. Hietanen and K. Rummukainen, Quark number susceptibility at high temperature, PoS LAT2006 (2006) 137 arXiv:hep-lat/0610111.

[27] A. Hietanen and K. Rummukainen, Quark number susceptibility of high temperature and finite density QCD, PoS LAT2007, 192 (2007) [arXiv:0710.5058 [hep-lat]].

[28] K. Kajantie, M. Laine, A. Rajantie, K. Rummukainen and M. Tsypin, The phase diagram of three-dimensional SU(3) + adjoint Higgs theory, JHEP 9811 (1998) 011 arXiv:hep-lat/9811004.

[29] A. Gynther, M. Laine, Y. Schroder, C. Torrero and A. Vuorinen, Four-loop pressure of massless $O(N)$ scalar field theory, JHEP 0704 (2007) 094 arXiv:hep-ph/0703307].

[30] M. Della Morte, R. Frezzotti, J. Heitger, J. Rolf, R. Sommer and U. Wolff [ALPHA Collaboration], Computation of the strong coupling in QCD with two dynamical flavours, Nucl. Phys. B 713 (2005) 378 [arXiv:hep-lat/0411025]. 
[31] V. G. Bornyakov, S. M. Morozov, Y. Nakamura, M. I. Polikarpov, G. Schierholz and T. Suzuki [DIK Collaboration], Finite temperature Lattice QCD with two flavors of improved Wilson fermions, PoS LAT2007 (2007) 171 [arXiv:0711.1427 [hep-lat]].

[32] M. Laine and A. Rajantie, Lattice-continuum relations for $3 d S U(N)+$ Higgs theories, Nucl. Phys. B 513, 471 (1998) arXiv:hep-lat/9705003.

[33] C. Torrero, M. Laine, Y. Schroder, F. Di Renzo and V. Miccio, Towards 4-loop NSPT result for a 3-dimensional condensate-contribution to hot QCD pressure, arXiv:0711.1176 [hep-lat]. 